\title{
LIMIT THEOREMS FOR SEMI-MARKOV PROCESSES $\left({ }^{1}\right)$
}

\author{
BY
}

JAMES YACKEL

1. Summary. For us a semi-Markov process is a separable process $\left\{X_{t}, t \geqq 0\right\}$ with denumerable state space $I$ such that if

$$
Y_{t}=\left\{\begin{array}{l}
t \quad \text { if } X_{s}=X_{t} \text { for all } 0 \leqq s \leqq t, \\
t-\sup \left[s: 0 \leqq s \leqq t, X_{s} \neq X_{t}\right] \quad \text { otherwise, }
\end{array}\right.
$$

then the two dimensional process $\left\{\left(X_{t}, Y_{t}\right) ; t \geqq 0\right\}$ is a strong Markov process with stationary Borel measurable transition probabilities. In this paper we are concerned with the limiting distributions of $X_{t}$ and $Y_{t}$.

In [8], Smith has shown that if the distributions which determine the time between jumps of the $X$ process (we refer to them as the waiting time distributions) have a finite first moment, then $X_{t}$ has a limiting distribution. His techniques depend on renewal theory results and are applicable to our processes. We extend the results for limiting distributions to include the possibility of instantaneous states. By showing that the joint limiting distribution of $X_{t}$ defines an invariant probability measure on the state space of the two dimensional Markov process we are able to find a limiting distribution for $Y_{t}$.

If the waiting time distributions fail to have a finite first moment we find conditions in $\$ 4$ which insure the existence of limit distributions for the $X$ process. Conversely, if limit distributions exist we find necessary conditions for the waiting time distributions. In $\$ 5$ the waiting time distributions are assumed to be in the domain of attraction of a stable law (c.f. [3]) in which case the conditions on the waiting time distributions, found in $\$ 4$, are shown to be both necessary and sufficient. Here, using results of Lamperti [6], we are able to find a joint limiting distribution for $X_{t}, Y_{t}$.

We conclude with an application to the construction of nonnormal Markov chains.

2. Notation and preliminaries. Denote the stationary Borel measurable transition probabilities by $p_{t}(i, y ; S)$ where $t>0,(i, y)$ is an element of the two di-

Received by the editors August 3, 1964 and, in revised form, January 10, 1966.

(1) This research was supported in part by the Air Force Office of Scientific Research and in part by a National Aeronautics and Space Administration Fellowship. This work is contained in the author's Ph.D. Thesis written under the direction of Professor Steven Orey. 
mensional state space of the $\left(X_{t}, Y_{t}\right)$ process and $S$ is a Borel subset of that state space, i.e. $p_{t}(i, y ; S)$ is a version of the conditional probability of the event $\left[\left(X_{t}, Y_{t}\right) \in S\right]$ given the initial state was $(i, y)$. For each point of the two dimensional state space $I \times[0, \infty), p .(i, y ; S)$ determines a measure on the events in the Borel field generated by $\left\{\left(X_{t}, Y_{t}\right), t \geqq 0\right\}$. We will denote that measure by $P_{(i, y)}[\cdot]$.

Let

$$
W_{t}=\left\{\begin{array}{l}
\inf \left[s: s \geqq t, X_{s} \neq X_{t}\right] \quad \text { if } X_{u}=X_{t} \text { for all } 0 \leqq u \leqq t, \\
\inf \left[s: s \geqq t, X_{s} \neq X_{t}\right]-\sup \left[s: s \leqq t, X_{s} \neq X t\right] \text { otherwise. }
\end{array}\right.
$$

$W_{t}$ is a random variable for each $t \geqq 0$ and we define for each $i \in I$,

$$
F_{i}(t)=P_{(i, 0)}\left[W_{0} \leqq t\right]
$$

the waiting time distribution function of the state $i$.

If $\lim _{t \rightarrow 0^{+}} F_{i}(t)=1$ we will say $i$ is an instantaneous state. For all noninstantaneous states $j$ (denote this set by $J$ ) we let

and

$$
0(j, 1)=\inf \left[t: t>0,\left(X_{t}, Y_{t}\right)=(j, 0)\right]
$$

$$
\theta(j, k)=\inf \left[t: t>\theta(j, k-1),\left(X_{t}, Y_{t}\right)=(j, 0)\right] \text { for } k>1 .
$$

Then we let $G_{i j}(t)=P_{(i, 0)}[\theta(j, 1) \leqq t]$ and form the iterated convolutions

$$
\begin{aligned}
& G_{i j}^{* 1}(t)=G_{i j}(t), \\
& G_{i j}^{* k}(t)=\int_{0}^{t} G_{i j}^{*(k-1)}(t-s) d G_{j j}(s), \quad k>1,
\end{aligned}
$$

for $i=j$ we define

$$
G_{j j}^{* 0}(t)=\left\{\begin{array}{l}
0 \text { if } t<0 \\
1 \text { if } t \geqq 0
\end{array}\right.
$$

As a regularity condition on the sample functions we ask that $X_{\theta(j, k)}=j$ a.s. (almost surely). Then the random variables $\theta(j, k), k=1,2,3, \cdots$ are Markov times of the process, see for instance [7]. Because the process has stationary transition probabilities and satisfies the strong Markov property, $0(j, k+1)-0(j, k)$ for $k=1,2,3, \cdots$ are a sequence of independent identically distributed random variables, each having the distribution $G_{j j}(\cdot)$.

The following is an immediate consequence of this observation.

Lemma 2.1. $P_{(i, 0)}[\theta(j, k) \leqq t]=G_{i j}^{* k}(t), k=1,2,3, \cdots$.

We now let $N(j, t)=\sup [k: \theta(j, k) \leqq t]$ and hence:

THEOREM 2.1. $\Sigma_{k=0}^{\infty} P_{(i, 0)}[N(j, t)=k]=1$ for all $j \in J$ and any $i \in I$. 
Proof. Since $\theta(j, k+1)-\theta(j, k), k=1,2,3, \cdots$ are nonnegative, independent and identically distributed random variables whose sum

$$
\sum_{k=1}^{n}[\theta(j, k+1)-\theta(j, k)]+\theta(j, 1)=\theta(j, n+1)
$$

and since $N(j, t) \geqq k$ if and only if $\theta(j, k) \leqq t$, it will suffice to demonstrate that the summands $\theta(j, k+1)-\theta(j, k)$ are not zero with probability 1 .

From the definition of $G_{j j}(\cdot)$ it is clear that $G_{j j}(\cdot) \leqq F_{j}(\cdot)$ and hence, $j \in J$ implies that the first moment of $G_{j j}(\cdot)$ is strictly positive. But $G_{j j}(\cdot)$ is the distribution of the summands.

Hence

with probability 1 .

$$
\sum_{k=1}^{\infty}[\theta(j, k+1)-\theta(j, k)]=\infty
$$

Given a $t \geqq 0$, for almost all $\omega$ there exists an integer $K(\omega)$ so that

$$
\sum_{k=1}^{K(\omega)}[\theta(j, k+1)-\theta(j, k)]>t
$$

which implies that $\theta(j, K(\omega))>t$ and hence $N(j, t) \leqq K(\omega)$. This completes the proof.

We let

and

$$
H_{i j}(t)=\sum_{k=1}^{\infty} G_{i j}^{* k}(t), \quad i \neq j
$$

$$
H_{j j}(t)=\sum_{k=0}^{\infty} G_{j j}^{* k}(t)
$$

Then $H_{i j}$ is a monotone increasing function, finite for each $t$. In what follows we will always assume that $J \neq \varnothing$ and that $\lim _{t \rightarrow \infty} G_{i j}(t)=1$ for all pairs $i, j \in I$.

We now find the distribution of $X_{t}$,

LEMMA 2.2 .

$$
P_{(i, 0)}\left[X_{t}=j, N(j, t)=k\right]=\int_{0}^{t}\left[1-F_{j}(t-s)\right] d G_{i j}^{* k}(s)
$$

for $k=1,2,3, \cdots$ and $j \in J$.

Proof. $\left[X_{t}=j, N(j, t)=k\right]=\left[X_{t}=j, W_{t}+\theta(j, k) \geqq , t \geqq \theta(j, k)\right]$ a.s. by the definition of the quantities involved.

Now the strong Markov property of the process tells us that

$$
P_{(i, 0)}\left[W_{\theta(j, k)} \leqq u \mid\left(X_{s}, Y_{s}\right) ; s \leqq \theta(j, k)\right]=P_{(j, 0)}\left[W_{\theta(j, k)} \leqq u\right]=F_{j}(u) .
$$

Hence $W_{\theta(j, k)}$ and $\theta(j, k)$ are independent random variables. Thus the distribution of the sum of $\theta(j, k)$ and $W_{\theta(j, k)}$ is given by the convolution of their dis- 
tribution functions, which is in this case, according to Lemma 2.1 and the above, given by

$$
\int_{0}^{t}\left[1-F_{j}(t-s)\right] d G_{i j}^{* k}(s)=P_{(i, 0)}\left[X_{t}=j, N(j, t)=k\right]
$$

as asserted.

THEOREM 2.2 .

$$
P_{(i, 0)}\left[X_{t}=j\right]=\int_{0}^{t}\left[1-F_{j}(t-s)\right] d I I_{i j}(s), \quad j \in J .
$$

Proof. According to Theorem 2.1,

$$
P_{(i, 0)}\left[X_{t}=j\right]=\sum_{k=1}^{\infty} P_{(i, 0)}\left[X_{t}=j, N(j, t)=k\right] .
$$

Lemma 2.2 gives each of these summands as $\int_{0}^{t}\left[1-F_{j}(t-s)\right] d G_{i j}^{* k}(s)$ and summing on $k$ yields the result.

3. Finite expected waiting times. We assume here that the first moment of the waiting time distribution is finite and that the distributions $G_{j j}$ are nonarithmetic, that is, there does not exist an $h>0$ so that $\Sigma_{k=0}^{\infty} G_{j j}(k h)=G_{j j}(\infty)$. We also assume that for each instantaneous state $i, \lim _{t \rightarrow 0^{+}} P_{(i, 0)}\left[X_{t}=i\right]=1$. If $\int_{0}^{\infty} s d F_{j}(s)=\mu_{j}$ and $\int_{0}^{\infty} s d G_{j j}(s)=\mu_{j j}$ then Smith [8] has shown that for all $j \in J$,

$$
\lim _{t \rightarrow \infty} P_{(i, 0)}\left[X_{t}=j\right]=\frac{\mu_{j}}{\mu_{j j}} .
$$

His proof applies to this more general semi-Markov process but we extend the result to include instantaneous states.

THeorem 3.1. If $j$ is an instantaneous state then $\lim _{t \rightarrow \infty} P_{(i, 0)}\left[X_{t}=j\right]$ exists and is independent of $i$.

Proof. Let $k \in J$, then

$$
P_{(i, 0)}\left[X_{t}=j\right]=P_{(i, 0)}\left[X_{t}=-j, N(k, t) \geqq 1\right]+P_{(i, 0)}\left[X_{t}=j, N(k, t)=0\right] .
$$

Since there is only one recurrent class,

$$
P_{(i, 0)}\left[X_{t}=j, N(k, t)=0\right] \leqq 1-G_{i k}(t) \rightarrow 0 \quad \text { as } t \rightarrow \infty .
$$

We treat the other term as follows,

$$
\begin{aligned}
P_{(i, 0)}\left[X_{t}=j, N(k, t) \geqq 1\right] & =\int_{0}^{t} P_{(i, 0)}\left[X_{t}=j, 0(k, 1) \in d s\right] \\
& =\int_{0}^{t} P_{(k, 0)}\left[X_{t-s}=j\right] P_{(i, 0)}[\theta(k, 1) \in d s]
\end{aligned}
$$

using the strong Markov property for $\theta(k, 1)$. 
By definition this is

$$
\int_{0}^{t} P_{(k, 0)}\left[X_{t-s}=j\right] d G_{i k}(s) .
$$

We now wish to show that for $k \in J, \lim _{t \rightarrow \infty} P_{(k, 0)}\left[X_{t}=j\right]$ exists for all instantaneous states $j$.

According to Theorem 2.1 we have

$$
\begin{aligned}
P_{(k, 0)}\left[X_{t}=j\right] & =\sum_{m=1}^{\infty} P_{(k, 0)}\left[X_{t}=j, N(k, t)=m\right] \\
& =\sum_{m=1}^{\infty} \int_{0}^{t} P_{(k, 0)}\left[X_{t}=j, \theta(k, m) \in d s, N(k, t)=m\right] .
\end{aligned}
$$

But using the strong Markov property for $\theta(k, m)$ we have

$$
\begin{aligned}
\sum_{m=1}^{\infty} \int_{0}^{t} P_{(k, 0)}\left[X_{t}=j, \theta(k, m) \in d s, N(k, t)=m\right] \\
\quad=\sum_{m=1}^{\infty} \int_{0}^{t} P_{(k, 0)}\left[X_{t-s}=j, N(k, t-s)=0\right] P_{(k, 0)}[\theta(k, m) \in d s] \\
=\sum_{m=1}^{\infty} \int_{0}^{t} P_{(k, 0)}\left[X_{t-s}=j, N(k, t-s)=0\right] d G_{k k}^{* m}(s) \\
=\int_{0}^{t} P_{(k, 0)}\left[X_{t-s}=j, N(k, t-s)=0\right] d H_{k k}(s) .
\end{aligned}
$$

If we can show that $P_{(k, 0)}\left[X_{t}=j, N(k, t)=0\right]$ is a Riemann integrable function satisfying the condition

$$
\sum_{k=0}^{\infty} \sup _{k \leqq t \leqq k+1}\left|P_{(k, 0)}\left[X_{t}=j, N(k, t)=0\right]\right|<\infty
$$

then an application of Blackwell's Renewal Theorem [1] to the convolution will give us the desired limit.

Note first that

$$
\lim _{t \rightarrow 0^{+}} P_{(j, 0)}\left[X_{t}=j, N(k, t)=0\right]=1
$$

if not $P_{(j, 0)}[N(k, t)>0] \geqq \delta>0$ for all $t>0$. Since we are assuming that $\lim _{t \rightarrow 0^{+}} P_{(j, 0)}\left[X_{t}=j\right]=1$ and because $N(k, t)>0$ means that $N(k, s)>0$ for all $s \geqq t$, if $P_{(j, 0)}[N(k, t)>0] \geqq \delta$ for all $t>0$ then $G_{j k}(t) \geqq \delta$ for all $t \geqq 0$ and thus from the representation of Theorem 2.2

$$
P_{(j, 0)}\left[X_{t}=k\right] \geqq \frac{\delta\left(1-F_{k}\left(0^{+}\right)\right)}{2}>0
$$


for all sufficiently small $t$, this contradicts the fact that $\lim _{t \rightarrow 0^{+}} P_{(j, 0)}\left[X_{t}=j\right]=1$.

Thus we see that

$$
\begin{aligned}
P_{(k, 0)}\left[X_{t+h}\right. & =j, N(k, t+h)=0] \mathrm{l} \\
& \geqq P_{(k, 0)}\left[X_{t}=j, N(k, t)=0\right] P_{(j, 0)}\left[X_{h}=j, N(k, h)=0\right] .
\end{aligned}
$$

Hence, from this and from (1) above, given any $\varepsilon>0$ there exists a $\delta>0$ and for all $h$ such that $0 \leqq h \leqq \delta$,

$$
P_{(k, 0)}\left[X_{t+h}=j, N(k, t+h)=0\right] \geqq P_{(k, 0)}\left[X_{t}=j, N(k, t)=0\right](1-\varepsilon) .
$$

Now $P_{(k, 0)}\left[X_{t}=j, N(k, t)=0\right]$ is a nonnegative function and

$$
P_{(k, 0)}\left[X_{t}=j, N(k, t)=0\right] \leqq 1-G_{k k}(t)
$$

where $1-G_{k k}$ is integrable, so if we can show that the discontinuities of the function are at most denumerable, $P_{(k, 0)}\left[X_{t}=j, N(k, t)=0\right]$ will satisfy the conditions for the Renewal Theorem.

We state the following lemma without proof.

LEMMA. If $f(x)$ is a nonnegative bounded function such that for any $\varepsilon>0$ there exists an $H>0$ independent of $x$ so that for all $0 \leqq h \leqq H, f(x+h) \geqq$ $f(x)(1-\varepsilon)$, then $f(x)$ has at most denumerably many discontinuities.

This shows the existence of the limit and proves Theorem 3.1.

We also state the result

THEOREM 3.2. For $j \in J$

$$
\lim _{t \rightarrow \infty} P_{(i, 0)}\left[X_{t}=j, Y_{t} \leqq \beta\right]=\frac{\int_{0}^{\beta}\left[1-F_{j}(s)\right] d s}{\mu_{j j}} .
$$

This theorem was known to Smith [8].

To show the existence of a limit distribution for $Y_{t}$ we must be able to perform an interchange of limits, i.e. we have the existence of

$$
\sum_{j \in I} \lim _{t \rightarrow \infty} P_{(i, 0)}\left[X_{t}=j, Y_{t} \leqq \beta\right]
$$

but we want to show the existence of

$$
\lim _{t \rightarrow \infty} P_{(i, 0)}\left[Y_{t} \leqq \beta\right]=\lim _{t \rightarrow \infty} \sum_{j \in I} P_{(i, 0)}\left[X_{t}=j, Y_{t} \leqq \beta\right]
$$

In order to justify the interchange of limits we will first show that we have a natural invariant measure which is a probability measure. That will yield the uniformity which allows the interchange of limits. 
The invariant measure is the measure induced on the state space by the joint limiting distribution of $X_{t}, Y_{t}$. We define the measure $\pi$

(i) for $i \in I, i \notin J$, let

$$
\pi[i \times[0, \beta]]=\lim _{s \rightarrow \infty} P_{(k, 0)}\left[X_{s}=i\right] ;
$$

(ii) for $j \in J$, let

$$
\pi[j \times[0, \beta]]=\frac{\int_{0}^{\beta}\left[1-F_{j}(s)\right] d s}{\mu_{j j}} .
$$

Cleary $\pi(\cdot)$ is a well defined measure.

We will let $Z$ denote the state space of $\left\{\left(X_{t}, Y_{t}\right), t \geqq 0\right\}$.

Lemma 3.1. $\pi(Z) \leqq 1$.

Proof. Clearly

$$
\sum_{j \in I} P_{(k, 0)}\left[X_{t}=j\right]=1
$$

and by Fatou's lemma

$$
\begin{aligned}
1=\liminf _{t \rightarrow \infty} \sum_{j \in I} P_{(k, 0)}\left[X_{t}=j\right] & \geqq \sum_{j \in I} \lim _{t \rightarrow \infty} P_{(k, 0)}\left[X_{t}=j\right] \\
& =\sum_{j \in I} \pi(j x[0, \infty))=\pi(Z),
\end{aligned}
$$

this completes the proof.

LEMMA 3.2. $\pi$ is an invariant measure.

Proof. The Chapman-Kolmogorov equation for the process has the form:

$$
\mathrm{P}_{(k, 0)}\left[\left(X_{t+s}, Y_{t+s}\right) \in B\right]=\int_{\mathrm{z}} P_{(z)}\left[\left(X_{t}, Y_{t}\right) \in B\right] P_{(k, 0)}\left[\left(X_{s}, Y_{s}\right) \in d z\right]
$$

where $z \in Z$ and $B \in B_{*}(Z)$ the Borel field of measurable $Z$ sets, and $0<s, t$. For $B \in B_{*}(Z)$, we will use the notation $B_{j}=[(j, t):(j, t) \in B]$.

$$
P_{(k, 0)}\left[\left(X_{t}, Y_{t}\right) \in B\right]=\underset{j \in I}{\sum_{(k, 0)}}\left[\left(X_{t}, Y_{t}\right) \in B_{j}\right]
$$

hence by Fatou

$$
\begin{aligned}
\liminf _{t \rightarrow \infty} P_{(k, 0)}\left[\left(X_{t}, Y_{t}\right) \in B\right] & \geqq \sum_{j \in I} \lim _{t \rightarrow \infty} P_{(k, 0)}\left[\left(X_{t}, Y_{t}\right) \in i B_{j}\right] \\
& =\sum_{j \in I} \pi\left(B_{j}\right)=\pi(B) .
\end{aligned}
$$


Thus since any bounded, nonnegative measurable function $g$ can be uniformly approximated by simple functions,

$$
\liminf _{t \rightarrow \infty} \int_{z} g(z) P_{(k, 0)}\left[\left(X_{t}, Y_{t}\right) \in d z\right] \geqq \int_{Z} g(z) \pi(d z) .
$$

Hence from the Chapman-Kolmogorov equation, for any $B \in B_{*}(Z)$, since $P_{(z)}[\cdot]$ is bounded, nonnegative and measurable,

$$
\begin{aligned}
\pi(B) & =\sum_{j \in I} \lim _{t \rightarrow \infty} P_{(k, 0)}\left[\left(X_{t+s}, Y_{t+s}\right) \in B_{j}\right] \\
& =\sum_{j \in I} \lim _{t \rightarrow \infty} \int_{Z} P_{(z)}\left[\left(X_{s}, Y_{s}\right) \in B_{j}\right] P_{(k, 0)}\left[\left(X_{t}, Y_{t}\right) \in d z\right] \\
& \geqq \sum_{J \in I} \int_{Z} P_{(z)}\left[\left(X_{s}, Y_{s}\right) \in B_{j}\right] \pi(d z) \\
& =\int_{Z} P_{(z)}\left[\left(X_{s}, Y_{s}\right) \in B\right] \pi(d z) .
\end{aligned}
$$

Suppose there is a $B \in B_{*}(Z)$ and an $s$ such that $\pi(B)>\int_{Z} P_{(z)}\left[\left(X_{s}, Y_{s}\right) \in B\right] \pi(d z)$.

Let $B^{\prime}$ denote the complement of $B$. Then

$$
\begin{aligned}
\pi(Z) & =\pi(B)+\pi\left(B^{\prime}\right)>\int_{Z} P_{(z)}\left[\left(X_{s}, Y_{s}\right) \in B\right] \pi(d z)+\int_{Z} P_{(z)}\left[\left(X_{s}, Y_{s}\right) \in B^{\prime}\right] \pi(d z) \\
& =\int_{Z}\left\{P_{(z)}\left[\left(X, Y_{s}\right) \in B\right]+P_{(z)}\left[\left(X_{s}, Y_{s}\right) \in B^{\prime}\right]\right\} \pi(d z) \\
& =\int_{Z} 1 \pi(d z)=\pi(Z) .
\end{aligned}
$$

This is a contradiction. Thus

$$
\pi(B)=\int_{Z} P_{(z)}\left[\left(X_{s}, Y_{s}\right) \in B\right] \pi(d z) \text { for every } B \in B_{*}(Z) \text {, for all } s>0 .
$$

Therefore $\pi$ is an invariant measure.

LEMMA 3.3. $\pi(Z)=1$.

Proof. If we normalize $\pi$, call it $\pi^{\prime}$, then $\pi^{\prime}$ is an invariant probability measurc. By bounded convergence

$$
\pi^{\prime}\left(B_{j}\right)=\lim _{s \rightarrow \infty} \int_{Z} P_{(z)}\left[\left(X_{s}, Y_{s}\right) \in B_{j}\right] \pi^{\prime}(d z)=\int_{Z} \pi\left(B_{j}\right) \pi^{\prime}(d z)=\pi\left(B_{j}\right)
$$

for all $j \in I$ and hence $\pi=\pi^{\prime}$. Therefore $\pi(Z)=\pi^{\prime}(Z)=1$, as asserted.

THEOREM 3.2. $Y_{t}$ converges in distribution. 
Proof. Given $\varepsilon>0$, by Lemma 3.3 there exists a finite set $F \subseteq I$ so that

$$
1-\sum_{j \in F} \pi\left(Z_{j}\right)<\varepsilon / 2
$$

Let $N$ be the cardinal number of the set $F$. Then for each $j \in F$ there is a $T_{j}$ so that $t \geqq T_{j}$ implies

$$
\left|P_{(i, 0)}\left[X_{t}=j, Y_{t} \leqq \beta\right]-\pi(j x[0, \beta])\right|<\varepsilon / 4 N .
$$

There is also a $T_{0}$ so that for all $t \geqq T_{0}$

$$
\left|1-\sum_{j \in F} P_{(i, 0)}\left[X_{t}=j\right]\right| \leqq \varepsilon / 4 .
$$

Then for all $t \geqq \operatorname{Max}\left[T_{0} ; T_{j}, j \in F\right]$,

$$
\begin{aligned}
\mid \sum_{j \in I} P_{(i, 0)} & {\left[X_{t}=j, Y_{t} \leqq \beta\right]-\sum_{j \in I} \pi(j \times[0, \beta]) \mid } \\
\leqq & \left|\sum_{j \in F} P_{(i, 0)}\left[X_{t}=j, Y_{t} \leqq \beta\right]-\sum_{j \in F} \pi(j \times[0, \beta])\right| \\
& +\left|\sum_{j \in I-F} P_{(i, 0)}\left[X_{t}=j, Y_{t} \leqq \beta\right]\right|+\sum_{j \in I-F} \pi(j \times[0, \beta]) \\
& <\varepsilon / 4+\varepsilon / 4+\varepsilon / 2
\end{aligned}
$$

from (1), (2), and (3). Thus

$$
\lim _{t \rightarrow \infty} P_{(i, 0)}\left[Y_{t} \leqq \beta\right]=\sum_{j \in I} \pi(j x[0, \beta]) .
$$

4. Infinite expected waiting times. Our assumption is now that the first moment of the distribution $F_{i}$, for all $i \in I$, does not exist. Thus $\mu_{i i}=\infty$ for $i \in I$ and hence the Renewal Theorem tells us that $\lim _{t \rightarrow \infty}\left[H_{i i}(t+h)-H_{i i}(t)\right]=0$ for all $h \geqq 0$.

We state the following without proof.

LEMMA 4.1. For every distribution function $F$ on $[0, \infty)$ and every $C>0$,

$$
0 \leqq \sum_{k=0}^{\infty} \sup _{k \leqq x \leqq k+1}[F(x+C)-F(x)]<\infty .
$$

This means that $F(x+C)-F(x)$ which is of bounded variation is Riemann integrable and hence the Renewal Theorem and representation of Theorem 2.2 immediately yield

LEMMA 4.2.

$$
\lim _{t \rightarrow \infty}\left\{P_{(i, 0)}\left[X_{t+h}=j\right]-P_{(i, 0)}\left[X_{t}=j\right]\right\}=0
$$

uniformly for all $0 \leqq h \leqq H$ for all $H>0$ and all $i, j \in I$. 
This then implies

LEMMA 4.3.

$$
\lim _{t \rightarrow \infty}\left\{P_{(j, 0)}\left[X_{t}=j\right]-P_{(i, 0)}\left[X_{t}=j\right]\right\}=0 \text { for all } i, j \in I .
$$

Proof. From the strong Markov property at the Markov time $\theta(j, 1)$ we have

$$
P_{(i, 0)}\left[X_{t}=j\right]=\int_{0}^{t} P_{(j, 0)}\left[X_{t-s}=j\right] d G_{i j}(s) .
$$

Given $\varepsilon>0$, according to our assumption, there exists an $H>0$ so that $1-\varepsilon<G_{i j}(H) \leqq 1$ and by Lemma 4.2 there exists a $T>0$ so that

$$
\left|P_{(j, 0)}\left[X_{t+h}=j\right]-P_{(j, 0)}\left[X_{t}=j\right]\right|<\varepsilon
$$

whenever $t \geqq T$ and $0 \leqq h \leqq H$.

Thus for all $t \geqq T+H$, we have the following:

$$
\begin{aligned}
\mid P_{(j, 0)}\left[X_{t}=j\right]-P_{(i, 0)}\left[X_{t}\right. & =j]|=| P_{(j, 0)}\left[X_{t}=j\right]-\int_{0}^{t} P_{(j, 0)}\left[X_{t-s}=j\right] d G_{i j}(s) \mid \\
& \leqq\left|P_{(j, 0)}\left[X_{t}=j\right]-\int_{0}^{H} P_{(j, 0)}\left[X_{t-s}=j\right] d G_{i j}(s)\right|+\varepsilon \\
& \leqq\left|P_{(j, 0)}\left[X_{t}=j\right]-P_{(j, 0)}\left[X_{t}=j\right] G_{i j}(H)\right|+\varepsilon G_{i j}(H)+\varepsilon \\
& \leqq 3 \varepsilon .
\end{aligned}
$$

This concludes the proof.

DEFINITION 4.1. Let

$$
{ }_{i} G_{j k}(t)=P_{(j, 0)}\left[X_{u}=k, N(i, u)=0 \text { for some } u, 0 \leqq u \leqq t\right], \quad j \neq k .
$$

We think of this as the "taboo" probability of a visit to state $k$ before a visit to state $i$ up to time $t$ with the initial distribution concentrated at $j$.

DEFINITION 4.2. Let ${ }_{i} G_{i k}^{* 1}(s)={ }_{i} G_{i k}(s)$, and form the convolutions

$$
{ }_{i} G_{i k}^{* n}(s)=\int_{0}^{s}{ }_{i} G_{i k}^{*(n-1)}(s-t) d_{i} G_{k k}(t), \quad n>1,
$$

then we write ${ }_{i} H_{i k}(s)=\Sigma_{n=1}^{\infty} G_{i k}^{* n}(s)$.

LEMMA 4.4.

$$
P_{(i, 0)}\left[X_{t}=j\right]=\int_{0}^{t}\left\{\int_{0}^{t-s}\left[1-F_{j}(t-s-u)\right] d_{i} H_{i j}(u)\right\} d H_{i i}(s), \quad i \neq j .
$$

Proof. This is a straighforward application of the strong Markov property based on the last visit to the state $i$. 
Corollary 4.1.

$$
\sup _{0 \leqq t \leqq \infty} \int_{0}^{t}\left[1-F_{j}(t-s)\right] d H_{i i}(s)<\infty .
$$

Proof. Suppose the conclusion is false, then for every integer $n$, there exists a number $T_{n}>0$ so that

$$
\int_{0}^{T_{n}}\left[1-F_{\jmath}(t-s)\right] d H_{i i}(s)>n .
$$

Clearly $T_{n} \rightarrow \infty$ as $n \rightarrow \infty$.

Now there is a positive number $K$ and a positive number $T^{\prime}$ depending on $K$ so that ${ }_{i} H_{i j}\left(T^{\prime}\right)>K$. Consider then for $0 \leqq h \leqq T^{\prime}$,

$$
\begin{aligned}
& \left|\int_{0}^{t+h}\left[1-F_{j}(t+h-s)\right] d H_{i i}(s)-\int_{0}^{t}\left[1-F_{j}(t-s)\right] d H_{i i}(s)\right| \\
& \quad \leqq\left|\int_{0}^{t}\left[F_{j}(t-s)-F_{j}(t+h-s)\right] d H_{i i}(s)\right|+\int_{0}^{t+h} 1 d H_{i i}(s) .
\end{aligned}
$$

As in Lemma 4.2 the first quantity approaches zero as $t$ becomes infinite. The second expression also approaches zero as $t$ becomes infinite according to the Renewal Theorem. Hence for any $\varepsilon>0$, there exists a $T^{\prime \prime}$ so large that $t>T^{\prime \prime}$ implies

$$
\int_{0}^{t+h}\left[1-F_{j}(t+h-s)\right] d H_{i i}(s) \text { and } \int_{0}^{t}\left[1-F_{j}(t-s)\right] d H_{i i}(s)
$$

are within $\varepsilon$ in value for all $0 \leqq h \leqq T^{\prime}$. That means then that for $T_{n} \geqq T^{\prime \prime}$,

$$
\int_{0}^{T_{n}+h}\left[1-F_{j}\left(T_{n}+h-s\right)\right] d H_{i i}(s)>n-\varepsilon
$$

and consequently, for $t \geqq T_{n}+h+T^{\prime}$

$$
\int_{0}^{t} \int_{0}^{t-s}\left[1-F_{j}(t-s-u)\right] d H_{i i}(u) d_{i} H_{i j}(s)>(n-\varepsilon) K .
$$

But from Lemma 4.4 thus quantity is bounded, this this is a contradiction. Hence $\sup _{0 \leqq t \leqq \infty} \int_{0}^{t}\left[1-F_{j}(t-s)\right] d H_{i i}(s)<\infty$, as asserted.

Theorem 4.1. If $\left[1-F_{i}(s)\right]=o\left(\left[1-F_{j}(s)\right]\right)$ for some $j \in I$ then

$$
\lim _{t \rightarrow \infty} P_{(k, 0)}\left[X_{t}=i\right]=0
$$

forall $k \in I$.

Proof. Given $\varepsilon>0$, by Lemma 4.4 


$$
\begin{aligned}
P_{(j, 0)}\left[X_{t}=i\right] & =\int_{0}^{t}\left\{\int_{0}^{t-s}\left[1-F_{i}(t-s-u)\right] d_{j} H_{j i}(u)\right\} d H_{j j}(s) \\
& =\int_{0}^{t}\left\{\int_{0}^{t-s}\left[1-F_{i}(t-s-u)\right] d H_{j j}(u)\right\} d_{j} H_{j i}(s)
\end{aligned}
$$

after a change in the order of integration and a change of variable.

By our assumption, there exists an $S$ so that for $s \geqq S,\left[1-F_{i}(s)\right]<$ $(\varepsilon / 2)\left[1-F_{j}(s)\right]$, and since $\lim _{x \rightarrow \infty}\left[H_{j j}(x+s)-H_{j j}(x)\right]=0$, there is a $T>0$ so that if $x \geqq T, H_{j j}(x+S)-H_{j j}(x)<\varepsilon / 2$.

Thus for all $s \geqq S+T$

$$
\begin{aligned}
\int_{0}^{s}\left[1-F_{i}(s-u)\right] d H_{j j}(u)= & \int_{0}^{s-s}\left[1-F_{i}(s-u)\right] d H_{j j}(u) \\
& +\int_{s-s}^{s}\left[1-F_{i}(s-u)\right] d H_{j j}(u) \\
\leqq & (\varepsilon / 2) \int_{0}\left[1-F_{j}(s-u)\right] d H_{j j}(u)+\varepsilon / 2 \\
= & (\varepsilon / 2) P_{(j, 0)}\left[X_{s}=j\right]+\varepsilon / 2 \leqq \varepsilon .
\end{aligned}
$$

Then since $G_{j j}(\infty)=G_{j i}(\infty)=1$ we see that ${ }_{j} H_{j i}(\infty)<\infty$, so that for any $\varepsilon^{\prime}>0$, there exists $S_{1}$ so that for $s \geqq S_{1}$,

$$
\int_{0}^{s}\left[1-F_{i}(s-u)\right] d H_{j j}(u)<\left(\varepsilon^{\prime} / 2\right)_{j} H_{j i}(\infty)
$$

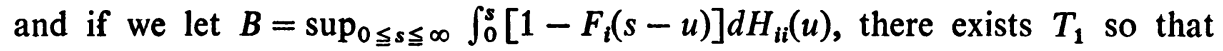
$t \geqq T_{1}$ implies ${ }_{j} H_{j i}(\infty)-{ }_{j} H_{j i}(t)<\varepsilon^{\prime} / 2 B$.

Then for $t \geqq S_{1}+T_{1}$

$$
\int_{0}^{t}\left\{\int_{0}^{t-s}\left[1-F_{i}(t-s-u)\right] d H_{j j}(u)\right\} d_{j} H_{j i}(s)<\varepsilon^{\prime} .
$$

Now by Lemma 4.3 the result follows.

THEOREM 4.2. If $\left[1-F_{i}(s)\right]-\lambda_{i j}\left[1-F_{j}(s)\right]=o\left(\left[1-F_{j}(s)\right]\right)+o\left(\left[1-F_{i}(s)\right]\right)$ $+g_{i j}(s)$ where $\Sigma_{k=0}^{\infty} \sup _{k \leqq s \leqq k+1}\left|g_{i j}(s)\right|<\infty$ then for $i \neq j$

$$
\lim _{t \rightarrow \infty}\left\{P_{(i, 0)}\left[X_{t}=i\right]-\left.\lambda_{i j}\right|_{i} H_{i j}(\infty) P_{(i, 0)}\left[X_{t}=j\right]\right\}=0 .
$$

Proof. Let $\varepsilon>0$ be given. If $\lambda_{i j}=0$ then we have $\left[1-F_{i}(s)\right]=o\left(\left[1-F_{j}(s)\right]\right)$ $+o\left(\left[1-F_{i}(s)\right]\right)+g_{i j}(s)$.

From Corollary 4.1,

so that

$$
\sup _{0 \leqq t<\infty} \int_{0}^{t}\left[1-F_{j}(t-s)\right] d H_{l i}(s)=K<\infty
$$




$$
\begin{array}{lll}
\lim _{t \rightarrow \infty} \int_{0}^{t} o\left(\left[1-F_{j}(t-s)\right]\right) d H_{i i}(s) & =0, \\
\lim _{t \rightarrow \infty} \int_{0}^{t} g_{i j}(t-s) d H_{i i}(s) & =0
\end{array}
$$

for any $g_{i j}$ satisfying the condition of this theorem and finally it is obvious that

$$
\lim _{t \rightarrow \infty} \int_{0}^{t} o\left(\left[1-F_{i}(t-s)\right]\right) d H_{i i}(s)=0 .
$$

Hence in this case the limit exists and is zero.

We assume then that $\lambda_{i j} \neq 0$. By Lemma 4.4 and Fubini's theorem we have

$$
P_{(i, 0)}\left[X_{t}=j\right]=\int_{0}^{t}\left\{\int_{0}^{t-s}\left[1-F_{j}(t-s-u)\right] d H_{i i}(u)\right\} d_{i} H_{i j}(s)
$$

We first consider

$$
\begin{aligned}
& \int_{0}^{t}\left[1-F_{i}(t-u)\right] d H_{i i}(u) \\
& =\int_{0}^{t}\left\{\lambda_{i j}\left[1-F_{j}(t-u)\right]+o\left(\left[1-F_{j}(t-u)\right]\right)+o\left(\left[1-F_{i}(t-u)\right]\right)+g_{i j}(t-u)\right\} d H_{i i}(u) .
\end{aligned}
$$

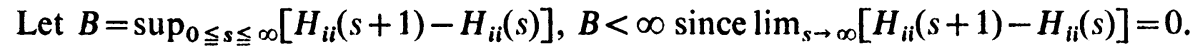
By assumption we can choose $T_{1}$ so that

$$
\sum_{k \geqq\left|T_{1}\right|} \sup _{k \leqq s \leqq k+1}\left|g_{i j}(s)\right|<\varepsilon / 4 B .
$$

Since $\left|g_{i j}\right|$ is bounded, let $B^{\prime}=\sup _{0 \leqq s \leqq \infty}\left|g_{i j}\right|$.

Now let $T_{2}$ be so chosen that $s \geqq T_{2}$ implies

$$
\left|o\left(\left[1-F_{j}(s)\right]\right)\right|<\varepsilon\left|1-F_{j}(s)\right| / 4 K .
$$

Similarly $o\left(\left[1-F_{j}(s)\right]\right)$ and $o\left(\left[1-F_{i}(s)\right]\right)$ are bounded so let

$$
B^{\prime \prime}=\sup _{0 \leqq s \leqq \infty}\left\{\left|o\left(\left[1-F_{j}(s)\right]\right)\right|+\left|o\left(\left[1-F_{i}(s)\right]\right)\right|\right\} .
$$

Obviously we can find $T_{3}$ so that $t \geqq T_{3}$ implies $\left|\int_{0}^{t} o\left(\left[1-F_{i}(t-s)\right]\right) d H_{i i}(s)\right|<\varepsilon / 4$. Now if $T_{4}=\operatorname{Max}\left(T_{1}, T_{2}, T_{3}\right)$ we choose $T_{5}$ so that if $t \geqq T_{5}$

$$
H_{i i}\left(T_{4}+t\right)-H_{i i}(t)<\varepsilon / 4\left(B^{\prime}+B^{\prime \prime}\right) .
$$

Then if $t \geqq T_{4}+T_{5}$, 


$$
\begin{aligned}
& \left|\int_{0}^{t}\left[1-F_{i}(t-u)\right] d H_{i i}(u)-\int_{0}^{t} \lambda_{i j}\left[1-F_{j}(t-u)\right] d H_{i i}(u)\right| \\
& \leqq \mid \int_{0}^{t-T_{4}}\left\{o\left(\left[1-F_{j}(t-u)\right]\right)+o\left(\left[1-F_{i}(t-u)\right]\right)+g_{i j}(t-u)\right\} d H_{i i}(u) \\
& +\int_{t-T_{4}}^{t}\left\{o\left(\left[1-F_{j}(t-u)\right]\right)+o\left(\left[1-F_{i}(t-u)\right]\right)+g_{i j}(t-u)\right\} d H_{i i}(u) \mid \\
& \leqq\left|\int_{0}^{t-T_{4}}\left\{o\left(\left[1-F_{j}(t-u)\right]\right)+o\left(\left[1-F_{i}(t-u)\right]\right)\right\} d H_{u}(u)\right| \\
& +\left|\int_{0}^{t-T_{4}} g_{i j}(t-u) d H_{i i}(u)\right| \\
& +\left|\int_{t-T_{4}}^{t}\left\{o\left(\left[1-F_{j}(t-u)\right]\right)+o\left(\left[1-F_{i}(t-u)\right]\right)+g_{i j}(t-u)\right\} d H_{i i}(u)\right| \\
& \leqq \varepsilon / 4 K\left|\int_{0}^{t-T_{4}}\left[1-F_{j}(t-u)\right] d H_{i i}(u)\right|+\left|\varepsilon / 4 \int_{0}^{t-T_{4}}\left[1-F_{i}(t-u)\right] d H_{i i}(u)\right| \\
& +\sum_{k=0}^{\left[t-T_{4}+1\right]} \sup _{k \leqq s \leqq k+1}\left|g_{i j}(t-s)\right|\left[H_{i i}(k+1)-H_{i i}(k)\right] \\
& +\left|B^{\prime}+B^{\prime \prime}\right|\left[H_{i i}(t)-H_{i i}\left(t-T_{3}\right)\right]<\varepsilon / 4+\varepsilon / 4+\varepsilon / 4+\varepsilon / 4 \text {. } \\
& \lim _{t \rightarrow \infty}\left\{\int_{0}^{t}\left[1-F_{i}(t-u)\right] d H_{i i}(u)-\lambda_{i j} \int_{0}^{t}\left[1-F_{j}(t-u)\right] d H_{i i}(u)\right\}=0 .
\end{aligned}
$$

Now by Lemma 4.2, and the integral representation we have,

$$
\lim _{t \rightarrow \infty}\left|\int_{0}^{t+h}\left[1-F_{i}(t+h-u)\right] d H_{i i}(u)-\int_{0}^{t}\left[1-F_{i}(t-u)\right] d H_{i i}(u)\right|=0 .
$$

Consequently we conclude that

$$
\begin{aligned}
& \lim _{t \rightarrow \infty}\left|\lambda_{i j} \int_{0}^{t}\left[1-F_{j}(t-u)\right] d H_{i i}(u)-\int_{0}^{t+h}\left[1-F_{i}(t+h-u)\right] d H_{i i}(u)\right| \\
& \quad \leqq \lim _{t \rightarrow \infty}\left|\lambda_{i j} \int_{0}^{t}\left[1-F_{j}(t-u)\right] d H_{i i}(u)-\int_{0}^{t}\left[1-F_{i}(t-u)\right] d H_{i i}(u)\right| \\
& \quad+\lim _{t \rightarrow \infty}\left|\int_{0}^{t}\left[1-F_{i}(t-u)\right] d H_{i i}(u)-\int_{0}^{t+h}\left[1-F_{i}(t+h-u)\right] d H_{i i}(u)\right|=0
\end{aligned}
$$

for all $0 \leqq h \leqq H$ for all $H>0$.

By Lemma 4.5 we can choose $H$ so that

$$
{ }_{i} H_{i j}(\infty)-{ }_{i} H_{i j}(H)<\varepsilon / \lambda_{i j} \sup _{0 \leqq s<\infty}\left\{\int_{0}^{s}\left[1-F_{j}(s-u)\right] d H_{i i}(u)\right\}
$$


and $T_{5}$ large enough that when $t \geqq T_{5}$ we have for all $0 \leqq h \leqq H$,

(v) $\left|\lambda_{i j} \int_{0}^{t}\left[1-F_{j}(t-u)\right] d H_{i i}(u)-\int_{0}^{t+h}\left[1-F_{i}(t+h-u)\right] d H_{i i}(u)\right|<\varepsilon /{ }_{i} H_{i j}(\infty)$.

Now for all $t \geqq T_{5}+H$,

$\left|\lambda_{i j} P_{(i, 0)}\left[X_{t}=j\right]-i H_{i j}(\infty) P_{(i, 0)}\left[X_{t}=i\right]\right|$

$=\left|\lambda_{i j} \int_{0}^{t}\left\{\int_{0}^{s}\left[1-F_{j}(s-u)\right] d H_{i i}(u)\right\} d_{i} H_{i j}(t-s)-{ }_{i} H_{i j}(\infty) \int_{0}^{t}\left[1-F_{i}(t-u)\right] d H_{i i}(u)\right|$

$\leqq\left|\lambda_{i j} \int_{t-H}^{t}\left\{\int_{0}^{s}\left[\varepsilon-F_{j}(s-u)\right] d H_{i i}(u)\right\} d_{i} H_{i j}(t-s)-{ }_{i} H_{i j}(\infty) \int_{0}^{t}\left[1-F_{j}(t-u)\right] d H_{i i}(u)\right|$

$+\left|\lambda_{i j} \int_{0}^{t-H}\left\{\int_{0}^{s}\left[1-F_{j}(s-u)\right] d H_{i i}(u)\right\} d_{i} H_{i j}(t-s)\right|$.

Now for $t-H \leqq s \leqq t$, by (v)

$$
\left|\lambda_{i j} \int_{0}^{s}\left[1-F_{j}(s-u)\right] d H_{i i}(u)-\int_{0}^{i}\left[1-F_{i}(s-u)\right] d H_{i u}(u)\right|<\varepsilon /{ }_{i} H_{i j}(\infty) .
$$

Hence

$$
\begin{aligned}
& \mid \lambda_{i j} \int_{t-H}^{t}\left\{\int_{0}^{s}\left[1-F_{j}(s-u)\right] d H_{i i}(u)\right\} d_{i} H_{i j}(t-s) \\
& \quad-{ }_{i} H_{i j}(\infty) \int_{0}^{t}\left[1-F_{i}(t-u)\right] d H_{i i}(u) \mid \\
& \leq \int_{t-H}^{t}\left\{\lambda_{i j} \int_{0}^{s}\left[1-F_{j}(s-u)\right] d H_{i i}(u)\right. \\
& \left.\quad-{ }_{i} H_{i j}(\infty) / H_{i} H_{i j}(H) \int_{0}^{t}\left[1-F_{i}(t-u)\right] d H_{i i}(u)\right\} d_{i} H_{i j}(t-s) \\
& \leqq{ }_{i} H_{i j}(H)\left[\varepsilon /{ }_{i} H_{i j}(\infty)+\varepsilon / H_{i j}(H)\right] \leqq 2 \varepsilon .
\end{aligned}
$$

Also by (iv),

$$
\left|\lambda_{i j} \int_{0}^{t-H}\left\{\int_{0}^{s}\left[1-F_{j}(s-u)\right] d H_{i i}(u)\right\} d_{i} H_{i j}(t-s)\right| \leqq \varepsilon .
$$

Hence since 8 is arbitrary

$$
\lim _{t \rightarrow \infty}\left\{\lambda_{i j} P_{(i, 0)}\left[X_{t}=j\right]-{ }_{i} H_{i j}(\infty) P_{(i, 0)}\left[X_{t}=i\right]\right\}=0 .
$$

Therefore since $\lambda_{i j} \neq 0$, the result follows. 
The condition of Theorem 4.2 is very nearly a condition on the ratios of the tails of the distributions, it is easily seen that one can define quantities $\lambda_{i}$, for $i \in I$ in such a way that if $\lambda_{j} \neq 0$, then $\lambda_{i j}=\lambda_{i} / \lambda_{J}$.

THEOREM 4.3. If I is a finite set and the hypotheses of Theorem 4.2 are satisfied for all $i, j \in I$ then

$$
\lim _{t \rightarrow \infty} P_{(i, 0)}\left[X_{t}=i\right]=\lambda_{i} /\left\{\lambda_{i}+\sum_{j \in I ; j \neq i} \lambda_{j}{ }_{i} H_{i j}(\infty)\right\} \text { for } i \in I .
$$

Proof. Let the cardinal number of $I$ be $N$. According to Theorem 4.2 we have for all $i, j \in I$ for which $\lambda_{i} \neq 0$,

$$
\lim _{t \rightarrow \infty}\left\{P_{(i, 0)}\left[X_{t}=i\right]-\left(\lambda_{i} / \lambda_{j i} H_{i j}(\infty)\right) P_{(i, 0)}\left[X_{t}=j\right]\right\}=0 .
$$

There exists $T$ large enough that if $t \geqq T, \lambda_{i} \neq 0$

$$
\left|\left(\lambda_{j i} H_{i j}(\infty) / \lambda_{i}\right) P_{(i, 0)}\left[X_{t}=i\right]-P_{(i, 0)}\left[X_{t}=j\right]\right|<\varepsilon / N
$$

if $\lambda_{j}=0$

$$
\left|P_{(i, 0)}\left[X_{t}=j\right]\right|<\varepsilon / N .
$$

Thus since $\boldsymbol{\Sigma}_{j \in I} P_{(i, 0)}\left[X_{t}=j\right]=1$ for all $t>0$,

$$
\left|\sum_{j \in I} P_{(i, 0)}\left[X_{t}=j\right]-P_{(i, 0)}\left[X_{t}=i\right]-\sum_{j \in I ; j \neq i}\left(\lambda_{j i} H_{i j}(\infty) / \lambda_{i}\right) P_{(i, 0)}\left[X_{t}=i\right]\right| \leqq \varepsilon .
$$

Thus the result is established.

We now state a corollary to Theorem 4.2.

Corollary 4.2. If $\Sigma_{j \in I} \lambda_{j i} H_{i j}(\infty)=\infty$ and the conditions of Theorem 4.2 are satisfied then

$$
\lim _{t \rightarrow \infty} P_{(i, 0)}\left[X_{t}=i\right]=0 .
$$

Proof. Given $0<\varepsilon<1$ we find a finite set $S$ so that $\left.\Sigma_{j \in S} \lambda_{j i} H_{i j}(\infty)\right)^{-1}<\varepsilon$. Let $N$ be the cardinal number of the set $S$.

For sufficiently large $t$, Theorem 4.2 says that

so that

$$
\left|\left(\lambda_{j i} H_{i j}(\infty) / \lambda_{i}\right) P_{(i, 0)}\left[X_{t}=i\right]-P_{(i, 0)}\left[X_{t}=j\right]\right|<\varepsilon / N
$$

$$
1 \geqq \sum_{j \in S} P_{(i, 0)}\left[X_{t}=j\right] \geqq \sum_{i \in S}\left(\lambda_{j i} H_{i j}(\infty) / \lambda_{i}\right) P_{(i, 0)}\left[X_{t}=i\right]-\varepsilon
$$

and by our choice of $S$ we have,

$$
P_{(i, 0)}\left[X_{t}=i\right] \leqq(1+\varepsilon) \lambda_{i} \varepsilon
$$

but $\varepsilon$ is arbitrary and the conclusion follows. 
Our best effort toward answering the question of necessary conditions is summarized by

TheOREM 4.4. If $\lim _{t \rightarrow \infty} P_{(i, 0)}\left[X_{t}=j\right]=\pi_{j}$ and $\lim _{t \rightarrow \infty} P_{(i, 0)}\left[X_{t}=i\right]=\pi_{i}$, then if $\pi_{j} \neq 0$

$$
\lim _{T \rightarrow \infty} \frac{\int_{0}^{T}\left[1-F_{i}(s)\right] d s}{\int_{0}^{T}\left[1-F_{j}(s)\right] d s}=\frac{\pi_{i i} H_{i j}(\infty)}{\pi_{j}} .
$$

Proof. Consider $\int_{0}^{T} P_{(i, 0)}\left[X_{s}=j\right]\left[1-F_{i}(T-s)\right] d s$ which equals

(i) $\int_{0}^{T}\left\{\int_{0}^{s}\left[\int_{0}^{s-u}\left[1-F_{j}(s-u-x)\right] d_{i} H_{i j}(x)\right] d H_{i i}(u)\right\}\left[1-F_{i}(T-s)\right] d s$

by Lemma 4.4. All integrands are nonnegative and bounded and all measures are finite on the range of integration, we apply Fubini's theorem to integrate on $s$ first. Hence

$$
\begin{aligned}
& \int_{0}^{T}\left\{\int_{u}^{T}\left[\int_{0}^{s-u}\left[1-F_{j}(s-u-x)\right]\left[1-F_{i}(T-s)\right] d_{i} H_{i j}(x)\right] d s\right\} d H_{i i}(u) \\
& \quad=\int_{0}^{T}\left\{\int_{0}^{T-u}\left[\int_{x+u}^{T}\left[1-F_{j}(s-u-x)\right]\left[1-F_{i}(T-s)\right] d s\right] d_{i} H_{i j}(x)\right\} d H_{i i}(u) .
\end{aligned}
$$

Now observe that this integral is symmetric in $\left[1-F_{j}\right]$ and $\left[1-F_{i}\right]$ so that we can interchange their roles in the original form of the expression found in (i). Therefore, (i) is equivalent to

$$
\int_{0}^{T}\left\{\int_{0}^{s}\left[\int_{0}^{s-u}\left[1-F_{i}(s-x-u)\right] d_{i} H_{i j}(x)\right] d H_{i i}(u)\right\}\left[1-F_{j}(T-s)\right] d s .
$$

Again by applying Fubini's theorem to interchange the two integrals with respect to ${ }_{i} H_{i j}$ and $H_{i i}$ measures, we get

$$
\begin{aligned}
& \int_{0}^{T}\left\{\int_{0}^{s}\left[\int_{0}^{s-x}\left[1-F_{i}(s-x-u)\right] d H_{i i}(u)\right] d_{i} H_{i j}(x)\right\}\left[1-F_{j}(T-s)\right] d s \\
& \quad=\int_{0}^{T}\left\{\int_{0}^{s} P_{(i, 0)}\left[X_{s-x}=i\right] d_{i} H_{i j}(x)\right\}\left[1-F_{j}(T-s)\right] d s \text { by Theorem 2.2. }
\end{aligned}
$$

Now by Lemma $4.5,{ }_{i} H_{i j}$ defines a finite measure on $[0, \infty)$ so that by the hypothesis that $\lim _{t \rightarrow \infty} P_{(i, 0)}\left[X_{t}=i\right]=\pi_{i}$ we see that

$$
\lim _{s \rightarrow \infty} \int_{0}^{s} P_{(i, 0)}\left[X_{s-x}=i\right] d_{i} H_{i j}(x)=\pi_{i}{ }_{i} H_{i j}(\infty) \text {. }
$$


Thus, given $\varepsilon>0$ there exists $T_{1}$ so that for $t \geqq T_{1}$ we have that

$$
{ }_{i} H_{i j}(\infty)-{ }_{i} H_{i j}(t)<\varepsilon,
$$

and there is a $T_{2}$ so that whenever $t \geqq T_{2}$.

$$
\left|P_{(i, 0)}\left[X_{t}=i\right]-\pi_{i}\right|<\varepsilon,
$$

hence for all $s \geqq T_{1}+T_{2}$,

$$
\left(\pi_{i}-\varepsilon\right)\left({ }_{i} H_{i j}(\infty)-\varepsilon\right)<\int_{0}^{s} P_{(i, 0)}\left[X_{s-x}=i\right] d_{i} H_{i j}(x)<\left(\pi_{i}+\varepsilon\right)\left({ }_{i} H_{i j}(\infty)+\varepsilon\right) .
$$

Let $L(s)=\int_{0}^{s} P_{(i, 0)}\left[X_{s-x}=i\right] d_{i} H_{i j}(x)$, then

(ii) $\lim _{s \rightarrow \infty} L(s)=\pi_{i i} H_{i j}(\infty)$,

(iii) $0 \leqq L(s) \leqq{ }_{i} H_{i j}(\infty)$

for all $0 \leqq s<\infty$.

We now write

$$
\int_{0}^{t} P_{(i, 0)}\left[X_{s}=j\right]\left[1-F_{i}(t-s)\right] d s=\int_{0}^{t} L(s)\left[1-F_{j}(t-s)\right] d s .
$$

All we need to show now is that only the tails of these integrals are important in the limit.

Let $\varepsilon^{\prime}>0$ be given so that $0<\varepsilon^{\prime}<\pi_{j}$, then by the hypothesis and from (ii) we can choose $T_{3}$ so that $s \geqq T_{3}$ implies $\left|P_{(i, 0)}\left[X_{s}=j\right]-\pi_{j}\right|<\varepsilon^{\prime}$ and

(v) $\left|L(s)-\pi_{i i} H_{i j}(\infty)\right|<\varepsilon^{\prime}$.

Now choose $T_{4}$ so that for all $T \geqq T_{4}$

(vi) $\varepsilon^{\prime} \cdot \int_{0}^{T}\left[1-F_{j}(s)\right] d s>T_{3}$.

This is possible by the assumption that the first moment of the distribution is infinite.

Then for all $t \geqq T_{3}+T_{4}$ and from (iii), (iv) and (v)

$$
\begin{aligned}
& \int_{0}^{T_{3}} P_{(i, 0)}\left[X_{s}=j\right]\left[1-F_{i}(t-s)\right] d s+\left(\pi_{j}-\varepsilon^{\prime}\right) \int_{T_{3}}^{t}\left[1-F_{i}(t-s)\right] d s \\
& \quad \leqq{ }_{i} H_{i j}(\infty) \int_{0}^{T_{3}}\left[1-F_{j}(t-s)\right] d s+\left(\pi_{i i} H_{i j}(\infty)+\varepsilon^{\prime}\right) \int_{T_{3}}^{t}\left[1-F_{j}(t-s)\right] d s .
\end{aligned}
$$

Now $0 \leqq 1-F_{i}(s) \leqq 1,0 \leqq 1-F_{j}(s) \leqq 1$ for all $s$ so that

$$
\begin{aligned}
\left(\pi_{j}-\varepsilon^{\prime}\right) & \left\{\int_{0}^{t}\left[1-F_{i}(s)\right] d s-T_{3}\right\} \\
& \leqq{ }_{i} H_{i j}(\infty) T_{3}+\left(\pi_{i i} H_{i j}(\infty)+\varepsilon^{\prime}\right) \int_{0}^{t}\left[1-F_{j}(s)\right] d s .
\end{aligned}
$$

Divide both sides of the above inequality by $\int_{0}^{t}\left[1-F_{j}(s)\right] d s$ and use (vi) to conclude 


$$
\left(\pi_{j}-\varepsilon^{\prime}\right) \frac{\int_{0}^{t}\left[1-F_{i}(s)\right] d s}{\int_{0}^{t}\left[1-F_{j}(s)\right] d s}-\varepsilon^{\prime} \leqq_{i} H_{i j}(\infty) \varepsilon^{\prime}+\left(\pi_{i i} H_{i j}(\infty)+\varepsilon^{\prime}\right) .
$$

The obvious symmetric argument yields the inequality,

$$
T_{3}+\left(\pi_{j}+\varepsilon^{\prime}\right) \int_{0}^{t}\left[1-F_{i}(s)\right] d s \geqq\left(\pi_{i i} H_{i j}(\infty)-\varepsilon^{\prime}\right)\left\{\int_{0}^{t}\left|1-F_{j}(s)\right| d s-T_{3}\right\}
$$

which, after we divide both sides by $\int_{0}^{t}\left[1-F_{j}(s)\right] d s$ and use (vi) as before, gives us the result.

\section{Waiting time distributions in the domain of attraction of a stable law.}

THEOREM 5.1. If $F_{i}$ and $F_{j}$ are in the domain of attraction of a stable law with exponent $\alpha$ and $\beta$ respectively where $0<\alpha, \beta<1$ and the respective Laplace transforms $1-\phi_{i}$ and $1-\phi_{j}$ satisfy the condition

then

$$
\lim _{\lambda \rightarrow 0^{+}} \frac{1-\phi_{i}(\lambda)}{1-\phi_{j}(\lambda)}=L \text { where } 0 \leqq L<\infty
$$

$$
\lim _{t \rightarrow \infty}\left\{P_{(i, 0)}\left[X_{t}=i\right]-\frac{L}{{ }_{i} H_{i j}(\infty)} P_{(i, 0)}\left[X_{t}=j\right]\right\}=0 .
$$

Proof. $F_{i}$ and $F_{j}$ being in the domain of attraction of a stable law implies that $1-F_{i}(s) \sim h_{i}(s) s^{-\alpha}$ and $1-F_{j}(s) \sim h_{j}(s) s^{-\beta}$ as $s \rightarrow \infty$ where $h_{i}$ and $h_{j}$ are slowly varying functions, i.e. $h$ is called slowly varying if $\lim _{t \rightarrow \infty} h(c t) / h(t)=1$ for all $c>0$, cf. [3, p. 175].

According to $[2, \mathrm{p} .460]$ our hypotheses imply $\lim _{t \rightarrow \infty}\left[1-F_{i}(t)\right] /\left[1-F_{j}(t)\right]=L$ and hence $\left[1-F_{i}(t)\right]=L\left[1-F_{j}(t)\right]+o\left(\left[1-F_{j}(t)\right]\right)$.

Thus if $L=0$ Theorem 4.1 implies the result. If $L \neq 0$ Theorem 4.2 implies the result.

Lemma 5.1 relies on the classical results of Karamata [4] for functions of slow growth.

Lemma 5.1. For distributions $F_{i}, F_{j}$ in the domain of attraction of a stable law with exponent $\alpha, 0<\alpha<1$,

implies

$$
\lim _{t \rightarrow \infty} \frac{\int_{0}^{t}\left[1-F_{i}(s)\right] d s}{\int_{0}^{t}\left[1-F_{j}(s)\right] d s}=L
$$

$$
\lim _{t \rightarrow \infty} \frac{\left[1-F_{i}(t)\right]}{\left[1-F_{j}(t)\right]}=L
$$


Proof. The ratio of the Laplace transforms of $1-F_{i}$ and $1-F_{j}$ is the same as the ratio of the Laplace transforms of $\int_{0}^{t}\left[1-F_{i}(s)\right] d s$ and $\int_{0}^{t}\left[1-F_{j}(s)\right] d s$. Now by hypothesis, if $I_{k}(t)=\int_{0}^{t}\left[1-F_{k}(s)\right] d s$, then $\lim _{t \rightarrow \infty} I_{k}(t)=\infty, k=i, j$. If $\varepsilon>0$ is given, there is a $T$ so large that for all $t \geqq T$,

(1) $\left|I_{i}(t)-L I_{j}(t)\right| \leqq \varepsilon I_{j}(t)$.

Consider now

$$
\begin{aligned}
& \left|\int_{0}^{\infty} e^{-s t}\left\{I_{i}(t)-L I_{j}(t)\right\} d t\right| \leqq \int_{0}^{\infty} e^{-s t}\left|I_{i}(t)-L I_{j}(t)\right| d t \\
& \quad=\int_{0}^{T} e^{-s t}\left|I_{i}(t)-L I_{j}(t)\right| d t+\int_{T}^{\infty} e^{-s t}\left|I_{i}(t)-L I_{j}(t)\right| d t \\
& \quad \leqq \int_{0}^{T} e^{-s t}\left|I_{i}(t)-L I_{j}(t)\right| d t+\varepsilon \int_{T}^{\infty} e^{-s t} I_{j}(t) d t
\end{aligned}
$$

using (1).

Since $I_{i}(t)$ and $I_{j}(t)$ are each bounded by $t$ for all $t \geqq 0$, the first term in (2) is bounded by $T^{2}(1+L)$ so that (2) is bounded above by

$$
T^{2}(1+L)+\varepsilon \int_{T}^{\infty} e^{-s t} I_{j}(t) d t \leqq T^{2}(1+L)+\varepsilon \int_{0}^{\infty} e^{-s t} I_{j}(t) d t
$$

But $\lim _{t \rightarrow \infty} I_{j}(t)=\infty$ so that for all $s>0, s$ sufficiently small we have

$$
\frac{T^{2}(1+L)}{\int_{0}^{\infty} e^{-s t} I_{j}(t) d t}<\varepsilon
$$

Thus dividing by $\int_{0}^{\infty} e^{-s t} I_{j}(t) d t$ in (2) we have

$$
\lim _{s \rightarrow 0^{+}}+\frac{\int_{0}^{\infty} e^{-s t} I_{i}(t) d t}{\int_{0}^{\infty} e^{-s t} I_{j}(t) d t}=L .
$$

Thus

$$
\frac{\int_{0}^{\infty} e^{-s t} I_{i}(t) d t}{\int_{0}^{\infty} e^{-s t} I_{j}(t) d t}=\frac{\int_{0}^{\infty} e^{-s t}\left[1-F_{i}(t)\right] d t}{\int_{0}^{\infty} e^{-s t}\left[1-F_{j}(t)\right] d t} \text { for all } s
$$

hence the limits are equal. But the limit of the ratio of the Laplace transforms shows the limit of the ratio of the distributions.

Thus for this class of distributions the conditions of $\$ 4$ on the distributions are both necessary and sufficient. 
In this context we can also discuss the joint limiting distribution of $X_{t}, Y_{t}$.

Let $\theta^{\prime \prime}(j, t)=\theta(j, N(j, t)+1)$, i.e. $\theta^{\prime \prime}$ is the next arrival time to state $j$ after time $t$. The strong Markov property allows us to view $\theta^{\prime \prime}(j, t)$ as a sum of independent random variables so that

LEMMA 5.2.

$$
P_{(j, 0)}\left[\theta^{\prime \prime}(j, t) \geqq t+t u\right]=\int_{0}^{t}\left[1-G_{j j}(t+t u-s)\right] d H_{j j}(s)
$$

for all $u>0$.

Proof.

$$
\begin{aligned}
P_{(j, 0)}\left[\theta^{\prime \prime}(j, t)\right. & \geqq t+t u, N(j, t)=k] \\
& =\int_{0}^{t} P_{(j, 0)}\left[\theta^{\prime \prime}(j, t) \geqq t+t u, N(j, t)=k, \theta(j, k) \in d s\right] \\
& =\int_{0}^{t} P_{(j, 0)}\left[\theta^{\prime \prime}(j, t-s) \geqq t+t u-s, N(j, t-s)=0\right] P_{(j, 0)}[\theta(j, k) \in d s]
\end{aligned}
$$

by the strong Markov property of the process at the time $\theta(j, k)$.

Now

$$
P_{(j, 0)}\left[\theta^{\prime \prime}(j, t-s) \geqq t+t u-s, N(j, t-s)=0\right]=1-G_{j j}(t+t u-s)
$$

so that this can be written as,

But

$$
\int_{0}^{t}\left[1-G_{j j}(t+t u-s)\right] d G_{j j}^{* k}(s)
$$

$$
\begin{aligned}
P_{(j, 0)}\left[\theta^{\prime \prime}(j, t) \geqq t+t u\right]=\sum_{k=0}^{\infty} P_{(j, 0)}\left[\theta^{\prime \prime}(j, t) \geqq t+t u, N(j, t)=k\right] \\
=\sum_{k=0}^{\infty} \int_{0}^{t}\left[1-G_{j j}(t+t u-s)\right] d G_{j j}(s)=\int_{0}^{t}\left[1-G_{j j}(t+t u-s)\right] d H_{j j}(s) .
\end{aligned}
$$

\section{LEMMA 5.3.}

$$
P_{(j, 0)}\left[X_{t}=j, Y_{t} \geqq \beta t\right]=\int_{0}^{t-\beta t}\left[1-F_{j}(t-s)\right] d H_{j j}(s) \text { for } 0 \leqq \beta<1 .
$$

Proof. This is proven exactly as Theorem 2.2 with the added restriction on $Y_{t}$ causing no difficulty.

LEMMA 5.4. If $\lim _{t \rightarrow \infty} P_{(j, 0)}\left[X_{t}=j\right]=\pi_{j} \neq 0$ and if $1-F_{j}(t) \sim h_{j}(t) t^{-\alpha}$ where $0<\alpha<1$, then 


$$
1-G_{j j}(t) \sim h_{j j}(t) t^{-\alpha} \text { and } \lim _{t \rightarrow \infty} \frac{h_{j}(t)}{h_{j j}(t)}=\frac{1}{\pi_{j}} .
$$

Proof. From the representation $P_{(j, 0)}\left[X_{t}=j\right]=\int_{0}^{t}\left[1-F_{j}(t-s)\right] d H_{j j}(s)$ we see that

$$
\int_{0}^{\infty} e^{-\lambda t} P_{(j, 0)}\left[X_{t}=j\right] d t=\frac{\left[1-\phi_{j}(\lambda)\right]}{\lambda\left[1-G_{j j}(\lambda)\right]},
$$

where $\hat{G}_{j j}(\lambda)=\int_{0}^{\infty} e^{-\lambda t} d G_{j j}(t)$. This follows since $H_{j j}(s)=\Sigma_{k=0}^{\infty} G_{j j}^{* k}(s)$.

Now if $\lim _{t \rightarrow \infty} P_{(j, 0)}\left[X_{t}=j\right]=\pi_{j}$ then

and hence

$$
\int_{0}^{\infty} e^{-\lambda t} P_{(j, 0)}\left[X_{t}=j\right] d t \sim \frac{\pi_{j}}{\lambda} \text { as } \lambda \rightarrow 0^{+}
$$

Since

$$
\lim _{\lambda \rightarrow 0^{+}} \frac{1-\phi_{j}(\lambda)}{1-G_{j j}(\lambda)}=\pi_{j}
$$

$$
1-\phi_{j}(\lambda) \sim \Gamma(1-\alpha) h_{j}\left(\frac{1}{\lambda}\right) \lambda^{\alpha} \text { as } \lambda \rightarrow 0^{+}
$$

the result follows from known results for slowly varying functions.

Theorem 5.2. If $\lim _{t \rightarrow \infty} P_{(j, 0)}\left[X_{t}=j\right]=\pi_{j}$ and $1-F_{j}(t) \sim h_{j}(t) t^{-\alpha}, 0<\alpha<1$, then

$$
\lim _{t \rightarrow \infty} P_{(j, 0)}\left[X_{t}=j, Y_{t} \leqq \beta t\right]=\pi_{j} \frac{\sin (\pi \alpha)}{\pi} \int_{0}^{\beta /(1-\beta)} v^{-\alpha}(1+v)^{-1} d v
$$

Proof. By Lemma 5.2,

$$
P_{(j, 0)}\left[\theta^{\prime \prime}(j, s) \geqq s+s u\right]=\int_{0}^{s}\left[1-G_{j j}(s+s u-v)\right] d H_{j j}(v) .
$$

This is then a problem in summing independent random variables each with distribution function $G_{j j}$ and asking for the distribution of the "excess". This problem has been considered by Lamperti, [6], his result is,

$$
\lim _{s \rightarrow \infty} P_{(j, 0)}\left[\theta^{\prime \prime}(j, s)-s \geqq s u\right]=\int_{u}^{\infty} \frac{\sin (\pi \alpha)}{\pi} v^{-\alpha}(1+v)^{-1} d v
$$

for $u>0$, and $0<\alpha<1$.

Thus

$$
\begin{aligned}
\lim _{s \rightarrow \infty} P_{(j, 0)}\left[\theta^{\prime \prime}(j, s) \geqq s+s u\right] & =\lim _{s \rightarrow \infty} \int_{0}^{s}\left[1-G_{j j}(s+s u-v)\right] d H_{j j}(v) \\
& =\int_{u}^{\infty} \frac{\sin (\pi \alpha)}{\pi} v^{-\alpha}(1+v)^{-1} d v
\end{aligned}
$$


From Theorem 5.4, since $\left[1-F_{j}(t)\right]-\pi_{j}\left[1-G_{j j}(t)\right]=o\left(\left[1-G_{j j}(t)\right]\right)$ Theorem 4.2 applies, and we have

$$
\pi_{j} \lim _{s \rightarrow \infty} \int_{0}^{s}\left[1-G_{j j}(s+s u-v)\right] d H_{j j}(v)=\lim _{s \rightarrow \infty} \int_{0}^{s}\left[1-F_{j}(s+s u-v)\right] d H_{j j}(v) .
$$

Thus according to Lemma 5.3,

$$
\begin{aligned}
\lim _{s \rightarrow \infty} P_{(j, 0)}\left[X_{s+s u}=j, Y_{s+s u} \geqq s u\right] & =\lim _{s \rightarrow \infty} \int_{0}^{s}\left[1-F_{j}(s+s u-v)\right] d H_{j j}(v) \\
& =\pi_{j} \int_{u}^{\infty} \frac{\sin (\pi \alpha)}{\pi} v^{-\alpha}(1+v)^{-1} d v .
\end{aligned}
$$

Now let $s+s u=t$ and $\beta=u / 1+u$. The result then follows.

Application. We merely note here that these results can be used to construct nonnormal Markov chains. Take for instance a two state discrete parameter semi-Markov process $\left\{X_{n}\right\}$ for which $\lim _{n \rightarrow \infty} P_{(1,0)}\left[X_{n}=1\right]$ does not exist. This can be easily done according to $\S 5$ if we merely choose two distributions in the same domain of attraction in such a way that the ratio of the slowly varying functions does not have a limiting value.

Then

$$
P_{(1,0)}\left[X_{n}=1\right]=\sum_{k=0}^{n}\left\{P_{(1,0)}\left[X_{k}=1, Y_{k}=0\right]-P_{(1,0)}\left[X_{k}=2, Y_{k}=0\right]\right\}
$$

does not have a limit as $n \rightarrow \infty$.

It is easily verified that for the Markov chain $\left(X_{n}, Y_{n}\right)$ the invariant measure $v$ satisfies the condition $v(1,0)=v(2,0)$ so that one of the conditions for normality, see [5], will be easily seen to be violated.

\section{BIBLIOGRAOHY}

1. D. Blackwell, A renewal theorem, Duke Math. J. 15 (1948), 145-150.

2. G. Doetsch, Theorie der Laplace-Transformation, Handbuch der Laplace-Transformation, Band I, Birkhäuser, Basel, 1950.

3. B. V. Gnedenko and A. N. Kolmogorov, Limit distributions for sums of independent random variables, Addison-Wesley, Cambridge, Mass., 1954.

4. J. Karamata, Sur un mode de croissance régulière des fonctions, Mathematica (Roumanie) 4 (1930), 38-53.

5. J. G. Kemeny and J. L. Snell, Potentials for denumerable Markov chains, Bull. Amer. Math. Soc. 67 (1961), 120-122.

6. John Lamperti, Some limit theorems for stochastic processes, J. Math. Mech. 7 (1958), 433-450.

7. Michel Loève, Probability theory, Van Nostrand, Princeton, N. J., 1960.

8. W. L. Smith, Regenerative stochastic processes, Proc. Roy. Soc. Ser. A 232 (1955), 6-31.

UNIVERSITY OF MINNESOTA, MiNNEAPOLIS, MiNNESOTA 